\title{
GEOMETRIC CONSTRUCTION \\ OF THE CLASSICAL $r$-MATRIX \\ FOR THE ELLIPTIC CALOGERO-MOSER SYSTEM
}

\author{
GLEB E. ARUTYUNOV \\ Steklov Mathematical Institute \\ Vavilova 42, GSP-1, 117966, Moscow, Russia \\ E-mail: arut@class.mi.ras.ru
}

\begin{abstract}
By applying the Hamiltonian reduction technique we derive a matrix first order differential equation that yields the classical $r$-matrices of the elliptic (Euler-) Calogero-Moser systems as well as their degenerations.
\end{abstract}

1. Introduction. The $r$-matrix of the elliptic Calogero-Moser (CM) model was first found in $[1,2]$ by direct computations. In [3] the same $r$-matrix was obtained from the $r$-matrix of the Euler-Calogero-Moser (ECM) model by the Hamiltonian reduction. In this lecture we derive, following [4], a new matrix first order differential equation on an unknown linear operator $r$ acting on the space $\mathcal{F}$ of $\operatorname{sl}(n, \mathbf{C})$-valued functions on a torus $\Sigma_{\tau}$ with a modular parameter $\tau$ :

$$
X=[r(X), D]-k \bar{\partial} r(X)+Q(X),
$$

where $D$ and $Q$ are constant diagonal matrices, $X=X(z, \bar{z}) \in \mathcal{F}$, and $k$ is a number. We show that its solution $r$ obeying a specific boundary condition is precisely the $r$-matrix of the elliptic CM model with a spectral parameter. The trigonometric and rational $r$ matrices correspond to degenerations of this equation when $\Sigma_{\tau}$ degenerates into a circle and into a point respectively. We observe that choosing another boundary condition one also gets the $r$-matrix of the elliptic ECM model [3] and its degenerations. In this sense eq.(1) can be treated as the generating equation for the family of dynamical $r$-matrices related to the CM systems.

The main tool we use to deduce (1) is the Hamiltonian reduction technique ${ }^{1}$. A hamiltonian action of a group $G$ with a Lie algebra $\mathcal{G}$ on a symplectic manifold $\mathcal{P}$ gives rise to a moment map $\mu: \mathcal{P} \rightarrow \mathcal{G}^{*}, \mathcal{G}^{*}$ is the dual to $\mathcal{G}$ with respect to a pairing $<,>$. Let

1991 Mathematics Subject Classification: 58F07, 22E67.

The paper is in final form and no version of it will be published elsewhere.

${ }^{1}$ Our approach is inspired by the paper [7]. 
$\mathcal{J} \in \mathcal{G}^{*}$ and $G_{\mathcal{J}} \subset G$ be the isotropy subgroup of $\mathcal{J}$ under the coadjoint action. Then a quotient $\mathcal{P}_{\text {red }}=\mu^{-1}(\mathcal{J}) / G_{\mathcal{J}}$ admits under some natural assumptions a symplectic structure [5]. The rational and trigonometric CM models are obtained according to this scheme by reducing the geodesic motions on the cotangent bundles of semi-simple Lie groups [6]. Their $L$-operators arise as the matrix functions of coordinates on the reduced phase space $\mathcal{P}_{\text {red }}$, while the Lax representation - as the equation of motion on $\mathcal{P}_{\text {red }}$. As usual $r$-matrices are defined by computing the Poisson bracket of two $L$-operators and arranging it in the form $\left\{L_{1}, L_{2}\right\}=\left[r_{12}, L_{1}\right]-\left[r_{21}, L_{2}\right]$, where $L_{1}=L \otimes 1, L_{2}=1 \otimes L$. The computation of the Poisson bracket can be considerably simplified by using the Dirac bracket construction. According to [7], the Poisson bracket on the reduced phase space can be presented in the following convenient form

$$
\{f, h\}_{\text {red }}=\{f, h\}-<\mathcal{J},\left[V_{f}, V_{h}\right]>,
$$

where $f, h$ are functions on $\mathcal{P}$ whose restrictions on $\mu^{-1}(\mathcal{J})$ are $G_{\mathcal{J}}$-invariant and $V_{f}$ denotes the solution of $\left\langle\mathcal{J},\left[X, V_{f}\right]\right\rangle=\xi_{X} f$, where $\xi_{X}$ is the hamiltonian vector field generated by $X \in \mathcal{G}$.

The elliptic CM model with the spectral parameter can be obtained starting from an infinite-dimensional phase space. Namely, the Hamiltonian reduction procedure runs as follows $[8,9]$. The infinite-dimensional phase space $\mathcal{P}$ is parametrized by the set $p=(\phi, c ; A, k)$, where $\phi, A \in \mathcal{F}, c, k \in \mathbf{C}$ and is equipped with the standard Poisson (symplectic) structure:

$$
\{f, h\}=\int_{\Sigma_{\tau}} d \bar{z} d z \operatorname{tr}\left(\frac{\delta f}{\delta \phi} \frac{\delta h}{\delta A}-\frac{\delta f}{\delta A} \frac{\delta h}{\delta \phi}\right)+\frac{\delta f}{\delta c} \frac{\delta h}{\delta k}-\frac{\delta f}{\delta k} \frac{\delta h}{\delta c} .
$$

With a pairing

$$
<(A, k),(\phi, c)>=\int_{\Sigma_{\tau}} d \bar{z} d z \operatorname{tr}(A \phi)+k c
$$

$\mathcal{P}$ can be identified with the cotangent bundle over the centrally extended $s l$ current algebra $(\phi, c)$ on $\Sigma_{\tau}$. The current group $\Sigma_{\tau} \times S L(n)$ acts on $\mathcal{P}$ as

$$
\begin{gathered}
(\phi(z, \bar{z}), c) \rightarrow\left(f(z, \bar{z}) \phi(z, \bar{z}) f^{-1}(z, \bar{z}), \quad c+\int_{\Sigma_{\tau}} d \bar{\eta} d \eta \operatorname{tr} \phi A\right), \\
(A(z, \bar{z}), k) \rightarrow\left(f(z, \bar{z}) A(z, \bar{z}) f^{-1}(z, \bar{z})-k \bar{\partial} f(z, \bar{z}) f^{-1}(z, \bar{z}), \quad k\right) .
\end{gathered}
$$

This action preserves the Poisson structure (3). The moment map is fixed to be

$$
\mu(p)=k \bar{\partial} \phi+[A, \phi]=\mathcal{J}, \quad \mathcal{J}=\nu J \delta(z, \bar{z}),
$$

where $J$ denotes some element of the coadjoint $s l$ orbit to be specified later (Sec.3) and $\nu$ is a coupling constant. Explicitly, $\mathcal{P}_{\text {red }}$ is described by a pair $(L, D)$, where $D$ is a constant diagonal matrix and $L$ is a solution of (7) with $A=D$. $L$ appears to be the $L$-operator of the elliptic CM system.

2. Generating equation. To start with we note that generically an element $A \in \mathcal{F}$ is gauge equivalent to a complex constant diagonal matrix $D(A)$ [9], i.e.

$$
A=g(A) D(A) g(A)^{-1}-k \bar{\partial} g(A) g(A)^{-1} .
$$


This matrix is defined up to the action of the elliptic affine Weyl group. Indeed, the gauge transformation with $f(z)=e^{2 \pi i \frac{\bar{w} z-w \bar{z}}{\tau-\bar{\tau}}}$, where $w=\operatorname{diag}\left(s_{1}, \ldots, s_{n}\right), s_{i}=m_{i}+\tau n_{i}$, $m_{i}, n_{i} \in \mathbf{Z}, \operatorname{tr} w=0$ leads to the substitution $D(A) \rightarrow D(A)+\frac{2 \pi i k}{\tau-\bar{\tau}} w$. We fix $D(A)$ by choosing the fundamental Weyl chamber. The factor $g(A)$ is not uniquely defined. An element $\tilde{g}(A)=g(A) t$, where a diagonal matrix $t=t(z)$ is an entire function, also satisfies (8). Requiring $\tilde{g}(A)$ to be doubly periodic, we get that $t(z)$ is a constant matrix, i.e. an element of a maximal torus $T$ of $S L(n)$. We also normalize $g$ as $g(D)=1$.

Let us assign to any $X \in \mathcal{F}$ a function $F_{X}$ on $\mathcal{P}$ :

$$
F_{X}(p)=<\phi, g(A) X g(A)^{-1}>,
$$

where $g(A)$ is some solution of (8). According to the choice (7), $G_{\mathcal{J}}$ acting on the surface $\mu^{-1}(\mathcal{J})$ coincides with a group of smooth mappings $\left\{g: \Sigma_{\tau} \rightarrow S L(n), g(0) \in H\right\}$, where $H$ is the isotropy group of $J$. Now assume $J$ to be such that $H \cap T=0$. In this case there is no ambiguity in the choice of $g(A)$ for $A$ restricted to $\mu^{-1}(\mathcal{J})$, i.e. on this surface $F_{X}$ is well defined.

Functions $F_{X}, X \in \mathcal{F}$ are of interest due to their specific properties. The first one is that $F_{X}$ is invariant with respect to $(5)$,(6) with $g \in G_{\mathcal{J}}$, i.e. it can be viewed as a genuine function on the reduced phase space $\mathcal{P}_{\text {red }}$. The second property is that if we parametrize $\mathcal{P}_{\text {red }}$ by a pair $(L, D)$, then owing to the normalization condition $g(D)=1 F_{X}$ restricted to $\mathcal{P}_{\text {red }}$ coincides with a matrix element $\langle L, X\rangle$ of the $L$-operator. Thus, we can use (2) for $F_{X}$ and $F_{Y}$ to compute the Poisson bracket between the matrix elements of the $L$-operator. The calculations are straightforward. Let $f \circ A$ be a shorthand for the gauge transformation (6). Introducing a derivative $\nabla_{X} g(A)=\frac{d}{d t} g\left(e^{t X} \circ A\right)_{\left.\right|_{t=0}}$ of $g(A)$ at the point $A$ along an orbit of gauge transformations, we find how the hamiltonian vector field $\xi_{X}$ generated by $X$ acts on $F_{Z}$ :

$$
\begin{aligned}
& \xi_{X} F_{Z}=\frac{d}{d t}<e^{t X} \phi e^{-t X}, g\left(e^{t X} \circ A\right) Z g\left(e^{t X} \circ A\right)^{-1}>_{\left.\right|_{t=0}} \\
= & <\phi, g(A)\left[g(A)^{-1} \nabla_{X} g(A)-g(A)^{-1} X g(A), Z\right] g(A)^{-1}>,
\end{aligned}
$$

On $\mathcal{P}_{\text {red }}$ this formula takes the form $\xi_{X} F_{Z}=<L,\left[\nabla_{X} g(D)-X, Z\right]>$ and it provides the second term in (2):

$$
<\mathcal{J},\left[V_{F_{X}}, V_{F_{Y}}\right]>=<L,\left[\nabla_{V_{F_{X}}} g(D)-V_{F_{X}}, Y\right]>.
$$

To obtain the bracket $\left\{F_{X}, F_{Y}\right\}_{\text {red }}$ we first calculate the quantities $\frac{\delta F_{X}}{\delta A_{i j}(\eta, \bar{\eta})}$ and $\frac{\delta F_{X}}{\delta \phi_{i j}(\eta, \bar{\eta})}$ and after substituting them into (3), we combine $\left\{F_{X}, F_{Y}\right\}$ with (10) according to (2). In this way we prove that there exists a linear operator $r: \mathcal{F} \rightarrow \mathcal{F}$ defined by

$$
r(X)(z, \bar{z})=\sum_{i j} \int_{\Sigma_{\tau}} d \bar{\eta} d \eta X_{i j}(\eta, \bar{\eta}) \frac{\delta g(A)}{\delta A_{i j}(\eta, \bar{\eta})}(z, \bar{z})-\frac{1}{2}\left(\nabla_{V_{F_{X}}} g(D)-V_{F_{X}}\right)
$$

and such that the Poisson bracket on $\mathcal{P}_{\text {red }}$ acquires the form

$$
\left\{F_{X}, F_{Y}\right\}_{r e d}=<L,[r(X), Y]+[X, r(Y)]>.
$$

Note that formula (11) reminds the one derived in [7] for the $r$-matrix of the trigonometric CM system without a spectral parameter. 
In contrast to the Poisson bracket on $\mathcal{P}_{\text {red }}, r$-matrix (11) depends on the extension of $F_{X}$ in the vicinity of $\mu^{-1}(\mathcal{J})$. We extend $F_{X}$ in a way as to get the simplest form of $r$. To this end we assume that vector $J$ is such that $s l(n)$ decomposes into the direct sum $\mathcal{H} \oplus \mathcal{T} \oplus \mathcal{C}$, where $\mathcal{H}$ and $\mathcal{T}$ are the Lie algebras of $H$ and $T$ respectively and $\mathcal{C}$ is an orthogonal to $\mathcal{H} \oplus \mathcal{T}$ with respect to the Killing metric. We also assume that $\mathcal{T}$ and $\mathcal{C}$ are two Lagrangian subspaces of the nondegenerate two-form $\omega(X, Y)=J([X, Y])$ defined on $\mathcal{T} \oplus \mathcal{C}$. These restrictions on $J$ are similar to that in the finite-dimensional case $[6,7]$ and will be justified in the next section.

Recall that $g(A)(z, \bar{z})=e^{X(z, \bar{z})}$ is defined up to the right multiplication by an element of $T$. We remove this ambiguity by choosing $X(z, \bar{z})$ to obey a boundary condition $X(0) \in$ $\mathcal{H} \oplus \mathcal{C}$. In addition, if $A \in \mu^{-1}(\mathcal{J})$, then $X(0) \in \mathcal{H}$.

Having fixed $g(A)$, we find that $\nabla_{X} g(D)(z, \bar{z})=(P X)(z, \bar{z})$, where $P: \mathcal{F} \rightarrow \mathcal{F}$ is a unit operator at all points of $\Sigma_{\tau}$ except zero and it projects $X(0)$ on $\mathcal{H} \oplus \mathcal{C}$ parallel to $\mathcal{T}$. For $X$ such that $X(0) \in \mathcal{H} \oplus \mathcal{C}$ due to the singularity of $\mathcal{J}$ eq. (10) reduces to $0=<L,\left[\nabla_{X} g(D)-X, Y\right]>=J\left(\left[X(0), V_{F_{Y}}(0)\right]\right)$. With our choice of $J$ we conclude that $V_{F_{Y}}(0) \in \mathcal{H} \oplus \mathcal{C}$ for any $Y$. Hence, defining $g(A)$ as described above, we get from (11) the following $r$-matrix

$$
r(X)(z, \bar{z})=\sum_{i j} \int_{\Sigma_{\tau}} d \bar{\eta} d \eta X_{i j}(\eta, \bar{\eta}) \frac{\delta g(A)}{\delta A_{i j}(\eta, \bar{\eta})}(z, \bar{z})
$$

This formula has a transparent geometric meaning. Defining a time evolution of the field $A(t)$ as: $A(0)=D$ and $\left.\frac{d A}{d t}\right|_{t=0}=X$, one has $r(X)=\frac{d}{d t} g(A(t))_{\left.\right|_{t=0}}$. Since eq.(8) is valid for any $t$, i.e.

$$
A(t)=g(A)(t) D(A)(t) g(A)(t)^{-1}-k \bar{\partial} g(A)(t) g(A)(t)^{-1},
$$

we differentiate it with respect to $t$ and put $t=0$. The result is equation (1), where $Q=\frac{d}{d t} D_{\left.\right|_{t=0}}$. For any smooth function $X(z, \bar{z}) \in \mathcal{F}$ it has a unique solution $r(X)$ obeying the boundary condition $r(X)(0) \in \mathcal{H} \oplus \mathcal{C}$. From (1) we also read off that the $r$-matrix is dynamical $[10,11]$ since it depends on $D$ accumulating the coordinates on $\mathcal{P}_{\text {red }}$. We refer to (1) as to the factorization problem for $s l$ connection. Hence, by construction the $r$-matrix of the elliptic CM model is defined as a unique solution of the factorization problem for $s l$ connection obeying some specific boundary condition.

3. Elliptic $r$-matrix. In this section we show how to solve (1). To this end we first analyze an equation on $\Sigma_{\tau}$ :

$$
\bar{\partial} \mathcal{E}(z, \bar{z})=\delta(z, \bar{z})
$$

In the vicinity of the origin eq.(14) defines a meromorphic function with a first order pole with the residue $1 / 2 \pi i$. We define a solution of (14) as a meromorphic function having simple poles at the points of the period lattice $\mathbf{Z} \tau_{1}+\mathbf{Z} \tau_{2}\left(\tau_{1}=1, \tau_{2}=\tau\right)$ with residues $1 / 2 \pi i$ and satisfying the quasiperiodicity condition:

$$
\mathcal{E}\left(z+\tau_{k}\right)=\mathcal{E}(z)+C_{\tau_{k}},
$$

where $C_{\tau_{k}}$ are complex numbers. Note that $\mathcal{E}(z)$ can not be doubly periodic since there is no elliptic functions with first order poles. The difference of two solutions $\mathcal{E}$ and $\tilde{\mathcal{E}}$ of 
(14) is a holomorphic but non-periodic function $\psi$ (poles and residues of $\mathcal{E}$ 's coincide) with

$$
\psi\left(z+\tau_{k}\right)=\psi(z)+\delta_{k}, \quad \delta_{k}=C_{\tau_{k}}-\tilde{C}_{\tau_{k}} .
$$

Recall that the numbers $C_{k}$ are not arbitrary. They obey Legendre's identity $C_{1} \tau_{2}-$ $C_{2} \tau_{1}=1$, which originates from integrating $\mathcal{E}(z)$ around the pole at the origin [13]. Therefore, we get $\delta_{1} \tau_{2}-\delta_{2} \tau_{1}=0$. The only holomorphic function obeying (16) with $\delta_{k}$ constrained as above is $\psi(z)=\alpha z+\beta, \alpha=\delta_{1}, \beta \in \mathbf{C}$. Hence, any two solutions of (14) are related as $\tilde{\mathcal{E}}(z)=\mathcal{E}(z)+\alpha z+\beta$. The Weierstrass zeta-function $\zeta(z)=$ $\frac{1}{z}+\sum_{n, m \in \mathbf{Z}}\left(\frac{1}{z-\omega_{n m}}+\frac{1}{\omega_{n m}}+\frac{z}{\omega_{n m}^{2}}\right), \quad \omega_{n m}=n \tau_{1}+m \tau_{2}$, satisfies the properties listed above and therefore represents a peculiar solution of (14). Thus, we conclude that any meromorphic function $\mathcal{E}(z)$ with only simple poles at the points of the period lattice $\mathbf{Z} \tau_{1}+\mathbf{Z} \tau_{2}$ with the residues $1 / 2 \pi i$ and obeying (15) is of the form

$$
\mathcal{E}(z)=\frac{1}{2 \pi i} \zeta(z)+\alpha z+\beta .
$$

When $\beta=0$ these functions are odd $\mathcal{E}(-z)=-\mathcal{E}(z)$.

Introduce the notation:

$$
X=\sum_{i j} x_{i j}(z, \bar{z}) e_{i j}, \quad Y=r(X)=\sum_{i j} y_{i j}(z, \bar{z}) e_{i j}, \quad D=\sum_{i} d_{i} e_{i i}, \quad Q=\sum_{i} q_{i} e_{i i}
$$

where $e_{i j}$ are the matrix unities, then eq.(1) is equivalent to the system

$$
\begin{aligned}
\bar{\partial} y_{i i} & =\frac{1}{k} t_{i} \\
\bar{\partial} y_{i j}+\frac{d_{i j}}{k} y_{i j} & =-\frac{1}{k} x_{i j}, \quad i \neq j,
\end{aligned}
$$

where $t_{i}(z, \bar{z})=q_{i}-x_{i i}(z, \bar{z})$ and $d_{i j}=d_{i}-d_{j}$.

By forming the convolution of the fundamental solution $\mathcal{E}(z)$ with the r.h.s. of (19) we restore the diagonal part $Y_{\text {diag }}$ of $Y$ up to a constant matrix $h \in \mathcal{T}: Y_{\text {diag }}(z, \bar{z})=$ $\sum_{i} \int_{\Sigma_{\tau}} \frac{d \bar{\eta} d \eta}{k} \mathcal{E}(z-\eta) t_{i}(\eta, \bar{\eta})+h$. Requiring $Y$ to be doubly periodic, we determine the unknown matrix $Q$ :

$$
Q=\frac{1}{2 i \Sigma_{\tau}} \int_{\Sigma_{\tau}} d \bar{\eta} d \eta \quad x_{i i}(\eta, \bar{\eta}) e_{i i}
$$

To solve eq.(20) by a similar device we need a doubly periodic solutions $\mathcal{E}_{i j}$ of the equation

$$
\bar{\partial} \mathcal{E}_{i j}(z, \bar{z})+\frac{d_{i j}}{k} \mathcal{E}_{i j}(z, \bar{z})=\delta(z, \bar{z}), \quad i \neq j .
$$

Eq.(22) coincides in essence with the one defining the $L$-operator of the elliptic CM model and it has a unique doubly periodic solution given by [9]

$$
\mathcal{E}_{i j}(z, \bar{z})=\frac{1}{2 \pi i} e^{\frac{d_{i j}}{k}(z-\bar{z})} \frac{\sigma\left(z+\frac{d_{i j}}{\pi k} \operatorname{Im} \tau\right)}{\sigma(z) \sigma\left(\frac{d_{i j}}{\pi k} \operatorname{Im} \tau\right)} \equiv w_{i j}(-z,-\bar{z}),
$$

where $\sigma(z)$ is the Weierstrass sigma-function. 
Combining all the pieces together we can write a general solution $Y(z)$ of eq.(1):

$$
Y=h+\int_{\Sigma_{\tau}} \frac{d \bar{\eta} d \eta}{k}\left(\mathcal{E}(z-\eta) \sum_{i} t_{i}(\eta, \bar{\eta}) e_{i i}-\sum_{i \neq j} w_{i j}(\eta-z, \bar{\eta}-\bar{z}) x_{i j}(\eta, \bar{\eta}) e_{i j}\right) .
$$

At this point we specify $J$. In the elliptic case [9] one should choose the following representative $J$ on the coadjoint $s l(n)$ orbit

$$
J=1-u \otimes s^{\dagger},
$$

where $u$ is a vector in $\mathbf{C}^{n}$ and $\bar{s}_{i}=1 / u_{i}$. Then eq.(7) defines the following $L$-operator:

$$
L=\sum_{i} p_{i} e_{i i}-\nu \sum_{i \neq j} \frac{u_{i}}{u_{j}} \mathcal{E}(z, \bar{z})_{i j} e_{i j} .
$$

The momentum part in $L$ follows from the diagonal part of (7) with $J_{i i}=0$. Later on we point out the connection of (26) with Krichever's $L$-operator [12].

The Lie algebra $\mathcal{H}$ of the isotropy group $H$ of $J$ is determined by $(X \in \mathcal{H})$ :

$$
u_{i}\left(s^{\dagger} X\right)_{j}-(X u)_{i} \overline{s_{j}}=0 .
$$

Choosing in $(27) i=j$, one gets $\left(s^{\dagger} X\right)_{i}=\frac{\bar{s}_{i}}{u_{i}}(X u)_{i}$ and thereby (27) reduces to $\frac{(X u)_{i}}{u_{i}}=$ $\frac{(X u)_{j}}{u_{j}}=\lambda$, where $\lambda \in \mathbf{C}$. One also has $\left(s^{\dagger} X\right)_{i}=\frac{\overline{s_{i}}}{u_{i}} \lambda u_{i}=\lambda \bar{s}_{i}$. Thus, we find

$$
\mathcal{H}=\left\{X \in \operatorname{sl}(n, \mathbf{C}): \quad X u=\lambda u, \quad s^{\dagger} X=\lambda s^{\dagger}, \quad \lambda \in \mathbf{C}\right\} .
$$

From (28) we can read off that $\mathcal{H} \cap \mathcal{T}=0$. Since the real dimension of $\operatorname{sl}(n, \mathbf{C})$ is $2\left(n^{2}-1\right)$ and $\mathcal{H}$ is defined by $4 n-4$ equations, we get $\operatorname{dim} \mathcal{H}=2\left(n^{2}-1\right)-(4 n-4)=2(n-1)^{2}$.

Decompose $s l(n)$ into the direct sum $\mathcal{H} \oplus \mathcal{T} \oplus \mathcal{C}$, as above. To describe $\mathcal{C}$ explicitly we introduce a matrix $C$

$$
C=z \otimes s^{\dagger}-u \otimes y^{\dagger}
$$

depending on two vectors $z, y \in \mathbf{C}^{n}$. Let $X \in \mathcal{H}$ and $X u=s^{\dagger} X=0$, then $\operatorname{Tr}(X C)=0$ by the cyclic property of the trace. On the other hand, if $X \in \operatorname{sl}(n)$ we have $\operatorname{Tr}(X C)=$ $\sum_{i k}\left(z_{i} \overline{s_{k}}-u_{i} \overline{y_{k}}\right) x_{k i}$ and consequently for $C$ to be orthogonal to any $X=\left(x_{i} \delta_{i j}\right) \in \mathcal{T}$, we get $z_{i} \bar{s}_{i}-u_{i} \bar{y}_{i}=\beta$ for any $i$, where $\beta$ is arbitrary. Orthogonality of $\mathcal{H}$ and $C$ also implies: $0=\operatorname{Tr}(J C)=\beta n\left(1-\left\langle s^{\dagger}, u>\right)=\beta n(1-n)\right.$ that gives $\beta=0$. Thus, $C \in \mathcal{C}$ if $\overline{y_{i}}=\frac{\bar{s}_{i}}{u_{i}} z_{i}=\frac{1}{u_{i}^{2}} z_{i}$. We put $\sum_{i} \frac{z_{i}}{u_{i}}=0$ to have the correct $\operatorname{dimension}$ of $\mathcal{C}: \operatorname{dim} \mathcal{C}=2(n-1)$. Note also that $\mathcal{T}$ and $\mathcal{C}$ form a pair of complementary Lagrangian subspaces with respect to $\omega(X, Y)=<J,[X, Y]>$ defined on $\mathcal{T} \oplus \mathcal{C}$.

Now we find that $(C u)_{i}=n z_{i}$ and $\left(s^{\dagger} C\right)_{i}=-\frac{n}{u_{i}^{2}} z_{i}$. This allows us to describe the action of a generic element $X \in \mathcal{H} \oplus \mathcal{C}$ on $u$ and $s$ :

$$
\begin{aligned}
& (X u)_{i}=\lambda u_{i}+n z_{i}, \\
& \left(s^{\dagger} X\right)_{i}=\frac{\lambda}{u_{i}}-\frac{n z_{i}}{u_{i}^{2}} .
\end{aligned}
$$

Summing up the second lines in (29) and taking into account $\sum \frac{z_{i}}{u_{i}}=0$, we find $\lambda$ : $\lambda=\frac{1}{n} \sum_{i} \frac{(X u)_{i}}{u_{i}}$. Solving (29) for $z_{i}$, we arrive at 
Proposition 1. Let $X$ be an arbitrary element of $\mathcal{H} \oplus \mathcal{C}$. Then the following relation

$$
u_{i}\left(s^{\dagger} X\right)_{i}+(X u)_{i} \frac{1}{u_{i}}=\frac{2}{n} \sum_{j} \frac{(X u)_{j}}{u_{j}}
$$

is valid for any $i$.

We use Proposition 1 to fix an element $h$. To this end we require $Y(0)$ to be an element of $\mathcal{H} \oplus \mathcal{C}$. Then by substituting $Y(0)$ in (30) we completely determine $h$ :

$$
h=-\int_{\Sigma_{\tau}} \frac{d \bar{\eta} d \eta}{k} \mathcal{E}(-\eta) \sum_{i} t_{i} e_{i i}+\sum_{i \neq j} \int_{\Sigma_{\tau}} \frac{d \bar{\eta} d \eta}{2 k}\left(\frac{u_{j}}{u_{i}} w_{i j} x_{i j}+\frac{u_{i}}{u_{j}} w_{j i} x_{j i}\right)\left(e_{i i}-\frac{1}{n} I\right) .
$$

Thus, we arrive at

Proposition 2. There is a unique solution $Y(z, \bar{z})$ of eq.(1) obeying the boundary condition $Y(0) \in \mathcal{H} \oplus \mathcal{C}$ :

$$
\begin{aligned}
& Y(z, \bar{z})=\sum_{i} \int_{\Sigma_{\tau}} \frac{d \bar{\eta} d \eta}{k}(\mathcal{E}(z-\eta)-\mathcal{E}(-\eta))\left(q_{i}-x_{i i}(\eta, \bar{\eta})\right) e_{i i} \\
&-\sum_{i \neq j} \int_{\Sigma_{\tau}} \frac{d \bar{\eta} d \eta}{k} w_{i j}(\eta-z, \bar{\eta}-\bar{z}) x_{i j}(\eta, \bar{\eta}) e_{i j} \\
&+\sum_{i \neq j} \int_{\Sigma_{\tau}} \frac{d \bar{\eta} d \eta}{2 k}\left(\frac{u_{j}}{u_{i}} w_{i j}(\eta, \bar{\eta}) x_{i j}(\eta, \bar{\eta})+\frac{u_{i}}{u_{j}} w_{j i}(\eta, \bar{\eta}) x_{j i}(\eta, \bar{\eta})\right)\left(e_{i i}-\frac{1}{n} I\right)
\end{aligned}
$$

where $q_{i}$ and $w_{i j}$ are given by (21) and (23) respectively.

Using the explicit form $(17)$ of $\mathcal{E}(z)$ and taking into account (21) it is easy to reduce the first line in (31) to

$$
\int_{\Sigma_{\tau}} \frac{d \bar{\eta} d \eta}{2 i k}\left(\frac{\Phi(z, \bar{z})}{\Sigma_{\tau}}-\frac{(\zeta(z-\eta)+\zeta(\eta))}{\pi}\right) \sum_{i} x_{i i}(\eta, \bar{\eta}) e_{i i}
$$

where we have introduced a function $\Phi(z, \bar{z})=\int_{\Sigma_{\tau}} \frac{d \bar{\eta} d \eta}{2 \pi i}(\zeta(z-\eta)+\zeta(\eta))$. Hence, despite $\alpha z+\beta$ enters (17) the solution of (1) does not depend on it. We get from (31) the following

THEOREM 1. The r-matrix corresponding to L-operator (26) is the following $\operatorname{sl}(n) \otimes$ $\operatorname{sl}(n)$-valued function on $\Sigma_{\tau} \times \Sigma_{\tau}$

$$
\begin{gathered}
r(z, \eta)=\left(\frac{\Phi(z, \bar{z})}{2 i k \Sigma_{\tau}}-\frac{\zeta(z-\eta)+\zeta(\eta)}{2 \pi i k}\right) \sum_{i} e_{i i} \otimes e_{i i}-\frac{1}{k} \sum_{i \neq j} w_{i j}(\eta-z, \bar{\eta}-\bar{z}) e_{i j} \otimes e_{j i} \\
+\frac{1}{2 k} \sum_{i \neq j}\left(e_{i i}-\frac{1}{n} I\right) \otimes\left(\frac{u_{j}}{u_{i}} w_{i j}(\eta, \bar{\eta}) e_{j i}+\frac{u_{i}}{u_{j}} w_{j i}(\eta, \bar{\eta}) e_{i j}\right) .
\end{gathered}
$$

$L$-operator as well as $r$-matrix (32) depend on a vector $u \in \mathbf{C}^{n}$. However, by conjugating $L$ with the matrix $e^{U}, U_{i j}=u_{i} \delta_{i j}$ this dependence may be removed. The corresponding $r$-matrix is given by $(32)$ with all $u_{i}=1$.

Now it is the time to state a connection of (32) with the $r$-matrix of the elliptic CM model found in $[1,2]$. Without loss of generality we can assume that the integration domain $\Sigma_{\tau}$ has the vertices at the points $\pm \frac{1}{2} \pm \frac{\tau}{2}$. Then by the oddness of $\zeta$-function one 
has $\int_{\Sigma_{\tau}} d \eta d \bar{\eta} \zeta(\eta)=0$ and therefore $\Phi(z, \bar{z})$ reduces to

$$
\Phi(z, \bar{z})=\int_{\Sigma_{\tau}} \frac{d \bar{\eta} d \eta}{2 \pi i} \zeta(z-\eta) .
$$

Eq.(33) means that $\Phi(z, \bar{z})$ is a solution of the equation $\bar{\partial} \Phi(z, \bar{z})=1$, i.e. $\Phi(z, \bar{z})=$ $\bar{z}+f(z)$, where $f(z)$ is an entire function. The monodromy properties of $\zeta$ define the ones for $\Phi(z, \bar{z}): \Phi\left(z+\tau_{k}, \bar{z}+\overline{\tau_{k}}\right)=\Phi(z, \bar{z})+\frac{\Sigma_{\tau}}{\pi} C_{\tau_{k}}$. For $f(z)$ this gives

$$
f\left(z+\tau_{k}\right)-f(z)=\frac{\Sigma_{\tau}}{\pi} C_{\tau_{k}}-\bar{\tau}_{k}
$$

Following the same lines as above we conclude that the only entire function obeying (34) is $f(z)=\alpha z+\beta$ with $\alpha=\frac{C_{\tau} / \pi-\bar{\tau}}{\tau}$. The constant $\beta$ is equal to zero by the oddness of $\Phi(z)$. Thus, we get for $\Phi(z, \bar{z})$ the following explicit expression

$$
\Phi(z, \bar{z})=\bar{z}-z+\frac{C_{1}}{\pi} \operatorname{Im} \tau z .
$$

In [1] Krichever's $L$-operator [12]: $L^{K r}=\sum_{i} p_{i} e_{i i}-\frac{\nu}{2 \pi i} \sum_{i \neq j} G_{i j}(z) e_{i j}$, where $G_{i j}(z)=$ $2 \pi i e^{\frac{d_{i j}}{k} \Phi(z, \bar{z})} \mathcal{E}_{i j}(z, \bar{z})$, was used to find the corresponding $r$-matrix. We see that $L^{K r}$ is related to (26) by the similarity transformation $L^{K r}(z)=W(z, \bar{z}) L(z, \bar{z}) W(z, \bar{z})^{-1}$, with $W(z, \bar{z})=e^{\frac{D}{k} \Phi(z, \bar{z})}$. Calculating $\left\{W_{2}, L_{1}\right\}$ with the help of the canonically conjugated variables $\{P, D\}=\frac{1}{2 i \Sigma_{\tau}} \sum_{i} e_{i i} \otimes e_{i i}, P=\sum_{i} p_{i} e_{i i}$ on $\mathcal{P}_{r e d}$, we can show [4] that the $r$-matrix for $L^{K r}$ is just the one found in $[1,2]$.

4. Trigonometric $r$-matrix. Consider the cotangent bundle to the centrally extended current algebra $S^{1} \rightarrow s u(n)$. It is known [14] that reducing it by the action of the current group $S^{1} \rightarrow S U(n)$ and imposing the moment $\mathcal{J}=J \delta(\varphi)$ with $J=i \nu \sum_{i \neq j}\left(e_{i j}+e_{j i}\right)$ one left with the phase space $(L, D)$ of the trigonometric CM model, where $L$ is the corresponding $L$-operator:

$$
L(\varphi)=i \sum_{i} p_{i} e_{i i}+\frac{\nu}{2 k} \sum_{i \neq j} \frac{e^{\frac{i}{k} d_{i j}(\pi-\varphi)}}{\sin \frac{\pi}{k} d_{i j}} e_{i j},
$$

Retracing the same steps as in the elliptic case one gets the following equation on the $r$-matrix (see [4] for details)

$$
X=[Y, D]-k Y^{\prime}+Q, \quad X, Y \in S^{1} \times s u(n), \quad D, Q \in \mathcal{T},
$$

where this time $\mathcal{T}$ is a maximal torus of $s u(n)$. Clearly, eq.(37) may be viewed as a degeneration of (1) that corresponds to a degeneration of $\Sigma_{\tau}$ into a circle. In this section we solve (37) explicitly and thereby recover the $r$-matrix of the trigonometric CM model [11].

The root decomposition of $s u(n)$ elements $X, Y, D$ and $Q$ is given by (18) with coefficients obeying the unitary condition $x_{j i}=-x_{i j}^{*}$, etc. From (37) one finds two equations on diagonal and nondiagonal parts of $Y$ respectively. Imposing the periodicity condition: $Y(0)=Y(2 \pi)$, we reconstruct $Y$ up to an element $h \in \mathcal{T}$ :

$$
Y(\varphi)=h+\frac{1}{k} \sum_{i j} \int_{0}^{2 \pi} d \varphi^{\prime} w_{i j}\left(\varphi, \varphi^{\prime}\right) x_{i j}\left(\varphi^{\prime}\right) e_{i j},
$$


where

$$
w_{i j}\left(\varphi, \varphi^{\prime}\right)=\left(\frac{i e^{-\frac{i \pi d_{i j}}{k}}}{2 \sin \frac{\pi}{k} d_{i j}}-\theta\left(\varphi, \varphi^{\prime}\right)\right) e^{-\frac{i d_{i j}}{k}\left(\varphi-\varphi^{\prime}\right)}, \quad w_{i i}\left(\varphi, \varphi^{\prime}\right)=\frac{\varphi}{2 \pi}-\theta\left(\varphi, \varphi^{\prime}\right)
$$

and $\theta\left(\varphi, \varphi^{\prime}\right)$ is the Heviside function.

Since $s u(n)=\mathcal{H} \oplus \mathcal{T} \oplus \mathcal{C}$, where $\mathcal{H}$ is a maximal proper Lie subgroup of $s u(n)$ and $\mathcal{C}$ is an orthogonal to $\mathcal{H} \oplus \mathcal{T}$, just as in the elliptic case we can fix $h$ by requiring $Y(0)$ to be an element of $\mathcal{H} \oplus \mathcal{C}$. From the results of [7] it follows that this requirement is equivalent to the set of relations $(1 \leq i \leq n)$ :

$$
\sum_{j} \operatorname{Im} Y_{i j}(0)=\frac{1}{n} \sum_{i \neq j} \operatorname{Im} Y_{i j}(0) .
$$

Substituting $Y(0)$ in (40), one finds $h$ that makes (40) true:

$$
h=\frac{1}{2 k} \sum_{i \neq j} \int_{0}^{2 \pi} d \varphi^{\prime} w_{i j}\left(0, \varphi^{\prime}\right) x_{i j}\left(\varphi^{\prime}\right)\left(\left(\frac{1}{n}-e_{i i}\right)+\left(\frac{1}{n}-e_{j j}\right)\right) .
$$

Combining (38) and (41), we finally get

Proposition 3. Eq.(37) has a unique solution $Y(\varphi)$ obeying the constraint $Y(0) \in$ $\mathcal{H} \oplus \mathcal{C}$ and it is given by (38) and (41).

As a direct consequence of this lemma we get that $r$-matrix of the trigonometric $\mathrm{CM}$ system is the following function on $S^{1} \times S^{1}$

$$
\begin{aligned}
& r\left(\varphi, \varphi^{\prime}\right)=\frac{\varphi-\pi}{2 \pi k} \sum_{i} e_{i i} \otimes e_{i i}+\frac{i}{2 k} \sum_{i \neq j} \frac{\cos \frac{\pi d_{i j}}{k}}{\sin \frac{\pi d_{i j}}{k}} e^{-i \frac{d_{i j}}{k}\left(\varphi-\varphi^{\prime}\right)} e_{i j} \otimes e_{i j} \\
& -\frac{1}{2 k} \sum_{i \neq j}\left(e_{i i}-\frac{1}{n}\right) \otimes\left(\frac{e^{-i \frac{d_{i j}}{k}}}{1-e^{-\frac{2 \pi i d_{i j}}{k}}} e_{i j}-\frac{e^{i \frac{d_{i j}}{k}}}{e^{\frac{2 \pi i d_{i j}}{k}}} e_{j i}\right)+\frac{1}{2 k} s\left(\varphi, \varphi^{\prime}\right) .
\end{aligned}
$$

In (42) we have introduced a matrix $s$ :

$$
s\left(\varphi, \varphi^{\prime}\right)=\left(\sum_{i \neq j} e^{-\frac{i d_{i j}}{k}\left(\varphi-\varphi^{\prime}\right)} e_{i j} \otimes e_{j i}+\sum_{i} e_{i i} \otimes e_{i i}\right) \epsilon\left(\varphi-\varphi^{\prime}\right),
$$

where $\epsilon\left(\varphi-\varphi^{\prime}\right)=\left[1-2 \theta\left(\varphi-\varphi^{\prime}\right)\right]$ is the sign function.

By direct calculations [4] one can prove the following

Proposition 4. Matrix s leads to the trivial Poisson bracket on the reduced phase space, i.e. the following relation is satisfied

$$
\left[s_{12}\left(\varphi, \varphi^{\prime}\right), L(\varphi) \otimes I\right]-\left[s_{21}\left(\varphi^{\prime}, \varphi\right), I \otimes L\left(\varphi^{\prime}\right)\right]=0 .
$$

Corollary. $r$-matrix of the trigonometric CM model is given by $(42)$ with $s\left(\varphi, \varphi^{\prime}\right)$ $=0$.

On $\mathcal{P}_{\text {red }}$ the variables $(P, X)$ are canonically conjugated: $\left\{P_{1}, X_{2}\right\}=-\frac{1}{2 \pi} \sum_{i} e_{i i} \otimes e_{i i}$. $L$-operator (36) as well as $r$-matrix (42) depend on the parameter $\varphi$. However, this parameter may be removed by the similarity transformation $L \rightarrow \tilde{L}=Q(\varphi) L(\varphi) Q(\varphi)^{-1}$, where $Q(\varphi)=e^{-\frac{i}{k} X(\pi-\varphi)}$. One can easily show that the $r$-matrix corresponding to the 
$\tilde{L}$ coincides with the one first found in [11] and then derived in [7] by the Hamiltonian reduction applied to the cotangent bundle $T^{*} S L(n)$.

5. Concluding remarks. A few remarks are in order. Assuming in (24) $h=0$ and choosing $\mathcal{E}(z)=\frac{1}{2 \pi i} \zeta(z)$, we find a matrix

$$
r(z, \eta)=\left(\frac{\Phi(z, \bar{z})}{2 i k \Sigma_{\tau}}-\frac{\zeta(z-\eta)}{2 \pi i k}\right) \sum_{i} e_{i i} \otimes e_{i i}-\frac{1}{k} \sum_{i \neq j} w_{i j}(\eta-z, \bar{\eta}-\bar{z}) e_{i j} \otimes e_{j i}
$$

that turns out to be an $r$-matrix for the $L$-operator

$$
L=\sum_{i}\left(p_{i}-f_{i i} \zeta(z)\right) e_{i i}-\nu \sum_{i \neq j} \mathcal{E}_{i j}(z) f_{i j} e_{i j}
$$

of the elliptic ECM model containing the additional dynamical variables $f_{i j}$ [3]. Thus, eq.(1) also covers the ECM system being a spin extension of the CM model and gives a suggestion that the ECM $r$-matrix can be obtained by the Hamiltonian reduction.

If $\Sigma_{\tau}$ degenerates into a point, eq.(1) takes the form $X=[r(X), D]+Q$. One can easily show that it defines the $r$-matrices of the rational CM and ECM systems without spectral parameter.

Since eq.(1) is Lie-algebraic it hopefully may be used to find spectral-dependent $r$ matrices for CM models related to the other root systems. We also suppose that the study of possible deformations of eq.(1) is a good starting point to develop the $r$-matrix approach [15] to the Ruijsenaars systems [16] being relativistic extensions of the CM models.

Acknowledgements. The author is grateful to L. Chekhov, A. Gorsky, A. Marshakov, P. B. Medvedev, S.Frolov for useful discussions. This work is supported in part by the RFFI grant.

\section{References}

[1] E. K. Sklyanin, Alg. Anal., 6(2) (1994) 227.

[2] H. W. Braden, T. Suzuki, Lett. Math. Phys. 30 (1994) 147.

[3] E. Billey, J. Avan, O. Babelon, Phys. Lett. A 188 (1994) 263.

[4] G. E. Arutyunov, P. B. Medvedev, Geometric construction of the classical $r$-matrix for the elliptic and trigonometric Calogero-Moser systems, preprint, hep-th/9511070.

[5] V. I. Arnol'd, Mathematical methods of classical mechanics, Graduate Texts in Math., 60, Spinger-Verlag, Berlin, New-York, 1989.

[6] M. A. Olshanetsky, A. M. Perelomov, Invent. Math. 37 (1976) 93; Phys. Reps. 71 (1981) 313; Phys. Reps. 94 (1983) 6;

D. Kazhdan, B. Kostant, S. Sternberg, Comm. Pure Appl. Math. 31 (1978) 481.

[7] J. Avan, O. Babelon, M. Talon, Alg. Anal., 6(2) (1994) 67.

[8] E. Markman, Comp. Math. 93 (1994) 255.

[9] A. Gorsky, N. Nekrasov, Elliptic Calogero-Moser system from two-dimensional current algebra, preprint, hep-th/9401021.

[10] O. Babelon, C. M. Viallet, Phys. Lett. B 237 (1989) 411.

[11] J. Avan, M. Talon, Phys. Lett. B 303 (1993) 33. 
[12] I. Krichever, Funk. Anal. Appl., 14 (1980) 45.

[13] N. I. Akhiezer, Elements of theory of elliptic functions, in Russian.

[14] A. Gorsky, N. Nekrasov, Nucl. Phys. B414 (1994), 213; B436 (1995), 582;

A. Gorsky, Integrable many body systems in the field theories, preprint UUITP-16/94, (1994).

[15] J. Avan and G. Rollet, The classical r-matrix for the relativistic Ruijsenaars-Schneider system, preprint BROWN-HET-1014 (1995).

[16] S. N. Ruijsenaars, Comm. Math. Phys. 110 (1987) 191. 